\title{
Pressão arterial em crianças portadoras de doença falciforme
}

\author{
Blood pressure in children with sickle cell disease
}

Ho Chi Hsien¹, João Thomas A. Carvalhaes², Josefina Aparecida P. Braga ${ }^{3}$

\section{RESUMO}

Objetivo: Avaliar os valores da pressão arterial (PA) em crianças portadoras de doença falciforme (DF).

Métodos: Estudo observacional unicêntrico descritivo de 70 crianças portadoras de DF. Os valores da PA obtidos foram classificados conforme as V Diretrizes Brasileiras de Hipertensão Arterial. Os pacientes foram distribuídos segundo o genótipo em grupo HbSS e HbSC e segundo a faixa etária: grupo I (três anos a quatro anos e 11 meses), grupo II (cinco anos a oito anos e 11 meses) e grupo III (nove anos a 13 anos e 11 meses). $\mathrm{Na}$ análise estatística, aplicou-se o teste $t$ de Student e a ANOVA, sendo significante $p<0,05$.

Resultados: A média e o desvio padrão (DP) das medidas da PA sistólica (PAS) ( $\mathrm{mmHg}$ ) foram 95,9 $\pm 11,45$ e da PA

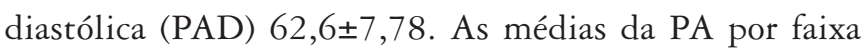

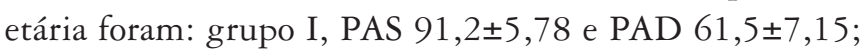

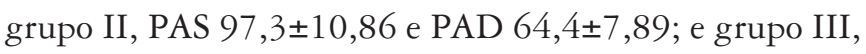
PAS $100,0 \pm 9,88$ e PAD $61,5 \pm 4,94$. Observou-se que $5,7 \%$ dos pacientes apresentavam hipertensão arterial (HA) e 8,6\% eram pré-hipertensos. A média dos valores da PAS e PAD entre os pacientes HbSS e HbSC não diferiu.

Conclusões: Novos estudos devem ser realizados para avaliar PA em pacientes com DF e detectar as possíveis causas de HA nesses pacientes.

Palavras-chave: pressão arterial; hipertensão; anemia falciforme; criança.

\section{ABSTRACT}

Objective: To evaluate blood pressure (BP) in children with sickle cell disease (SCD).

Methods: Observational descriptive study of BP in 70 children with SCD. BP values were classified according to the $\mathrm{V}$ Brazilian Guidelines in Arterial Hypertension. Patients were divided into groups according to genotype (HbSS, HbSC) and according to age: group I, three to four years and 11 months; group II, five to eight years and 11 months; and group III, nine to 13 years and 11 months. The Student's $t$ test and ANOVA were used for statistical analyses, and the level of significance was set at $p<0.05$.

Results: Mean and standard deviation (SD) of systolic BP (SBP) $(\mathrm{mmHg})$ were $95.9 \pm 11.45$, and of diastolic BP (DBP), 62.6 \pm 7.78. Means according to age group were: group I - SBP 91.2 \pm 5.78 and DBP 61.5 \pm 7.15 ; group II - SBP $97.3 \pm 10.86$ and DBP $64.4 \pm 7.89$; and group III

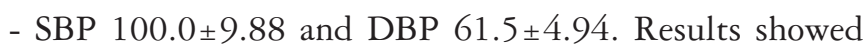
that $5.7 \%$ of the patients had hypertension and $8.6 \%$, pre-hypertension. Mean SBP and DBP of HbSC and HbSS patients did not differ.

Conclusions: Further studies should be conducted to assess BP in patients with SCD and determine possible causes of hypertension in these patients.

Key-words: blood pressure; hypertension; anemia, sickle cell; child.
Instituição: Escola Paulista de Medicina da Universidade Federal de São Paulo (Unifesp), São Paulo, SP, Brasil

${ }^{1}$ Mestre em Pediatria e Ciências Aplicadas à Pediatria pela Escola Paulista de Medicina da Unifesp; Médico Assistente do Setor de Nefrologia Pediátrica do Departamento de Pediatria da Escola Paulista de Medicina da Unifesp, São Paulo, SP, Brasil

${ }^{2}$ Doutor em Pediatria pela Escola Paulista de Medicina da Unifesp; Professor Adjunto e Chefe do Setor de Nefrologia Pediátrica do Departamento de Pediatria da Escola Paulista de Medicina da Unifesp, São Paulo, SP, Brasil ${ }^{3}$ Doutora em Pediatria e Ciências Aplicadas à Pediatria pela Escola Paulista de Medicina da Unifesp; Professora Adjunta e Chefe do Setor de Hematologia Pediátrica do Departamento de Pediatria da Escola Paulista de Medicina da Unifesp, São Paulo, SP, Brasil

\author{
Endereço para correspondência: \\ Josefina Aparecida P. Braga \\ Rua Dr. Diogo de Faria, 307 \\ CEP 04037-000 - São Paulo/SP \\ E-mail: pellegrini.braga@unifesp.br \\ Conflito de interesse: nada a declarar \\ Recebido em: 3/3/2011 \\ Aprovado em: 20/7/2011
}




\section{Introdução}

A doença falciforme (DF) é a doença hematológica hereditária mais frequente no Brasil, atingindo expressiva parcela da população em diferentes países. No Brasil, estima-se a ocorrência de 3.500 casos novos ao ano ${ }^{(1,2)}$. O gene da HbS pode se combinar com outras anormalidades hereditárias das hemoglobinas, como hemoglobina $\mathrm{C}$, hemoglobina $\mathrm{D}$ e $\beta$-talassemia, entre outros, gerando combinações que também são sintomáticas, denominadas, respectivamente de hemoglobinopatia SC, hemoglobinopatia SD e S/ $\beta$-talassemia. Todas essas formas sintomáticas do gene da HbS são conhecidas como doença falciforme $e^{(2)}$.

A DF apresenta aspectos epidemiológicos, manifestações clínicas e hematológicas variáveis de acordo com o genótipo, sendo a HbSS a forma mais grave da doença. Caracteriza-se por numerosas complicações, entre elas os episódios agudos de vaso-oclusão e a doença crônica, que pode afetar quase todos os órgãos e sistemas, determinando assim expressiva morbidade e redução da expectativa de vida desses pacientes ${ }^{(2)}$.

Alterações renais são complicações comuns na DF, iniciando-se na infância. Estima-se que 1/3 dos adolescentes e adultos jovens portadores de DF apresentem nefropatia. Dentre as anormalidades renais, são descritas a incapacidade de concentração urinária, o defeito na acidificação urinária e na excreção de potássio, além de distúrbios glomerulares, como hiperfiltração glomerular e proteinúria ${ }^{(3-5)}$. A detecção de hipertensão arterial (HA), embora frequente na etnia negra, tem se mostrado incomum nos pacientes portadores de anemia falciforme ${ }^{(3)}$. Estudos realizados em adultos portadores de DF demonstraram que estes apresentavam menor incidência de HA e nível de pressão arterial (PA) mais baixa em relação aos pacientes sem hemoglobinopatia $S$, mas tais achados não foram confirmados na população infantil ${ }^{(6-10)}$. Gordeuk et $a l^{(11)}$ observaram que os pacientes portadores de doença falciforme mostravam risco mais elevado de hipertensão pulmonar e insuficiência renal, quando os níveis pressóricos estavam aumentados.

A avaliação dos níveis pressóricos deve fazer parte da avaliação pediátrica, que já conta com valores pressóricos normatizados para crianças e adolescentes saudáveis ${ }^{(12)}$. No entanto, em crianças portadoras de DF, os níveis de PA têm sido pouco estudados. Dessa forma, o objetivo deste estudo foi avaliar os valores da PA em crianças e adolescentes com diagnóstico de DF, acompanhados no Setor de Hematologia Pediátrica da Universidade Federal de São Paulo/Escola Paulista de Medicina (Unifesp/EPM), com a finalidade de verificar se os valores pressóricos são semelhantes aos da população de crianças sem hemoglobinopatia e se a HA, que é um fator de risco para acidente vascular encefálico e hipertensão pulmonar no paciente portador de anemia falciforme, está presente nessa população.

\section{Método}

Estudo observacional unicêntrico, descritivo, desenvolvido no ambulatório de Hematologia Pediátrica do Hospital São Paulo da Unifesp/EPM no período de março de 2006 a julho de 2007. Foram incluídos todos os pacientes portadores de DF (hemoglobinopatias HbSS e HbSC) com idades de três a 14 anos, que compareceram ao ambulatório no período do estudo. Os pacientes foram distribuídos segundo o genótipo em grupo HbSS e HbSC e, segundo a faixa etária, em grupo I (três anos a quatro anos e 11 meses), grupo II (cinco anos a oito anos e 11 meses) e grupo III (nove anos a 13 anos e 11 meses). Foram excluídos pacientes portadores de hemoglobinopatia HbS $\beta$-talassemia e aqueles em uso crônico de corticosteróides, anti-inflamatórios, anticonvulsivantes, anti-histamínicos, bronco-dilatador, digitálico, hipotensores ou, que na ocasião da coleta de dados, apresentasse quadro febril ou crise de falcização.

O estudo foi aprovado pelo Comitê de Ética em Pesquisa da Unifesp. O consentimento expresso pós-informação foi obtido dos pais ou responsável.

As medidas da PA e do peso e altura foram obtidas sempre pelo mesmo pesquisador (pediatra nefrologista), no período da manhã, em formulário específico. Obteve-se três medidas da PA para cada paciente, em três ocasiões diferentes, sendo tomados todos os cuidados necessários para minimizar a ansiedade e o medo em relação ao procedimento. Utilizou-se esfigmomanômetro aneróide (Lane), adquirido junto à OPAS, com aferições periódicas, e os manguitos (Welch Allyn) de números seis, oito, dez e 12. A técnica da medida da PA seguiu a orientação da V Diretrizes Brasileiras de Hipertensão Arterial ${ }^{(12)}$. Os valores PA obtidos no grupo de pacientes foram comparados com os valores de PA já estabelecidos na literatura e definidos na V Diretrizes Brasileiras de Hipertensão Arterial ${ }^{(12)}$. Assim, o valor de PA sistólica (PAS) e diastólica (PAD) foi definido como normal quando inferior ao valor do percentil 90 para idade, sexo e percentil de estatura, desde que inferior a 120/80mmHg. O termo limítrofe foi definido a partir de valores de PA iguais ou superiores ao percentil 90 e inferiores ao percentil 95 para idade, sexo e percentil 
de estatura; para os adolescentes, qualquer valor igual ou superior a $120 / 80 \mathrm{mmHg}$ foi considerado limítrofe, mesmo sendo inferior ao percentil 95 para idade, sexo e percentil de estatura. A HA foi considerada quando os valores de PA foram iguais ou superiores ao percentil 95 para idade, sexo e percentil de estatura ${ }^{(12)}$.

Para obter o peso, utilizou-se balança de plataforma digital (Filizola ${ }^{\circledR}$ ) e, para a altura, estadiômetro vertical de madeira, cujo esquadro foi ajustado ao couro cabeludo em ângulo reto com o estadiômetro. As medidas antropométricas foram realizadas com o paciente descalço e o mínimo de roupa possível. Os dados antropométricos e a classificação do estado nutricional foram analisados com os programas Epi-Info versão 6.04b, Microsoft ${ }^{\circledR}$ Excel, Anthro $2006^{(13)} \mathrm{e}$ Anthro Plus $2007^{(14)}$ da OMS. Os índices antropométricos utilizados para avaliação do estado nutricional foram peso/ idade (P/I), estatura/idade (E/I), peso/estatura (P/E) medidos em escore Z. A classificação do estado nutricional, pelo IMC, foi realizada por meio das Curvas de Crescimento da Organização Mundial da Saúde (OMS) $)^{(13,14)}$.

As variáveis quantitativas foram descritas por medidas de tendência central, sendo comparadas pelo teste $t$ de Student. Na comparação das variáveis entre as diferentes faixas etárias, foi utilizado a ANOVA. Em todos os testes foi considerado um nível de significância de 5\% $(p<0,05)$.

\section{Resultados}

No período estudado foram incluídos 70 pacientes, sendo $35(50 \%)$ do gênero feminino; a idade variou de três anos e dois meses a 14 anos (média e desvio padrão de 7,2 $\pm 3,2$ anos e mediana de 6,1 anos). Quanto ao genótipo, 44 (63\%) pacientes eram HbSS e 26 (37\%) HbSC.

A média dos valores $(\mathrm{mmHg})$ e o desvio padrão (DP) da PAS e PAD foram, respectivamente, 95,9 $\pm 11,4$ e 62,6 $\pm 7,7$. Em relação aos genótipos HbSS e HbSC, obteve-se, respectivamente, 97,7 $\pm 10,1$ e 93,0 $0 \pm 8,4$ $(p=0,060)$ para PAS e $62,4 \pm 7,1$ e $62,53 \pm 8,7 \quad(p=0,460)$ para PAD.

O estado nutricional antropométrico piorou significantemente com a progressão da idade (Tabela 1). A desnutrição proteica energética (DPE) estava presente em cinco pacientes $(7,14 \%)$, sendo dois do grupo I e três grupo III. O risco de DPE ocorreu em $12(17,1 \%)$ pacientes e, destes, cinco pertenciam ao grupo I e sete ao grupo III.

Os valores da PAS aumentaram significantemente com progredir da faixa etária, não ocorrendo o mesmo com os valores da PAD (Tabela 2).

A HA foi constatada em quatro $(5,7 \%)$ pacientes, sendo dois do grupo I (um HbSC e um HbSS) e dois do grupo II (ambos HbSS), sem diferenças entre os sexos. PA limítrofe foi observada em seis $(8,6 \%)$ pacientes, destes um (HbSC) pertencia ao grupo I e cinco ao grupo II (quatro HbSS e um HbSC); os cinco pacientes eram do sexo masculino. Os quatro pacientes com HA e os seis pacientes com valores de PA limítrofe não apresentavam alterações significantes no perfil antropométrico em relação ao demais.

Ao comparar os valores da PAS e PAD obtidos no grupo de pacientes desnutridos e com risco para desnutrição em relação ao grupo de pacientes eutróficos, não se observou diferenças significantes nos valores da PA. (Tabelas 3 e 4).

Tabela 1 - Valores antropométricos dos pacientes portadores de doença falciforme, segundo o grupo etário

\begin{tabular}{lcccc}
\hline & Grupo I (n=24) & Grupo II $\mathbf{( n = 2 7 )}$ & Grupo III $\mathbf{( n = 1 9 )}$ & Valor $\boldsymbol{p}$ \\
\hline Estatura (cm) & & & & \\
Média & $103,6 \pm 5,5$ & $120,4 \pm 8,5$ & $138,8 \pm 7,7$ & $<0,001$ \\
Percentil & $60,0 \pm 24,6$ & $36,1 \pm 27,2$ & $20,8 \pm 22,5$ & $<0,001$ \\
Escore Z E/I & $0,24 \pm 1,29$ & $-0,22 \pm 0,74$ & $-1,41 \pm 1,05$ & $<0,001$ \\
Peso (kg) & & & & \\
Média & $16,4 \pm 2,3$ & $22,8 \pm 3,9$ & $30,8 \pm 7,0$ & $<0,001$ \\
Percentil & $52,7 \pm 29,0$ & $45,1 \pm 22,9$ & $13,2 \pm 18,4$ & $<0,001$ \\
Escore Z P/I & $0,04 \pm 0,92$ & $-0,12 \pm 0,70$ & $-1,68 \pm 1,29$ & $<0,001$ \\
Escore Z P/E & $-0,31 \pm 0,96$ & $0,11 \pm 0,47$ & - & $<0,001$ \\
IMC & & & & \\
Média & $15,5 \pm 1,3$ & $15,7 \pm 1,3$ & $15,8 \pm 11,9$ & 0,227 \\
Escore Z IMC & $-0,42 \pm 1,29$ & $-0,01 \pm 0,74$ & $-1,09 \pm 1,04$ & 0,012 \\
\hline
\end{tabular}

E/I: estatura/idade; P/I: peso/idade; IMC: índice de massa corpórea 
Tabela 2 - Medidas da pressão arterial sistólica, pressão arterial diastólica, nos pacientes portadores de doença falciforme, segundo o grupo etário

\begin{tabular}{lcccc}
\hline & Grupo I $(\mathbf{n}=\mathbf{2 4})$ & Grupo II $(\mathbf{n}=\mathbf{2 7})$ & Grupo III $\mathbf{( n = 1 9 )}$ & Valor $\boldsymbol{p}$ \\
\hline PAS $(\mathrm{mmHg})$ & & & & \\
Média $\pm \mathrm{DP}$ & $91,2 \pm 5,8$ & $97,3 \pm 10,9$ & $100,0 \pm 9,9$ & 0,006 \\
Mediana & 90 & 98 & 100 & \\
Q1 & 88 & 90 & 90 & \\
Q3 & 94 & 104 & 108 & \\
PAD (mmHg) & & & $61,5 \pm 4,9$ & 0,229 \\
Média \pm DP & $61,5 \pm 7,1$ & $64,4 \pm 7,9$ & 60 & \\
Mediana & 60 & 63 & 58 & \\
Q1 & 56 & 60 & 64 & \\
Q3 & 66 & 70 & & \\
\hline
\end{tabular}

PAS: pressão arterial sistólica; PAD: pressão arterial diastólica; DP: desvio padrão; Q1: $1^{\circ}$ quartil; Q3: $3^{\circ}$ quartil

Tabela 3 - Média das medidas da pressão arterial sistólica nos pacientes portadores de doença falciforme, segundo o estado nutricional e o grupo etário

\begin{tabular}{lccccc}
\hline & Eutróficos & $\mathbf{n}$ & Risco DPE & $\mathbf{n}$ & Valor $\boldsymbol{p}$ \\
\hline Grupo I & $91,1 \pm 5,8$ & 20 & $90,4 \pm 3,2$ & 04 & 0,283 \\
Grupo II & $97,3 \pm 10,8$ & 22 & - & 02 & NA \\
Grupo III & $99,3 \pm 10,0$ & 09 & $100,1 \pm 7,8$ & 10 & 0,386 \\
\hline
\end{tabular}

NA: não analisável; DPE: desnutrição proteico energética

Tabela 4 - Média das medidas da pressão arterial diastólica, nos pacientes portadores de doença falciforme, segundo o estado nutricional e o grupo etário

\begin{tabular}{lccccc}
\hline & Eutróficos & $\mathbf{n}$ & Risco DPE & $\mathbf{n}$ & Valor $\boldsymbol{p}$ \\
\hline Grupo I & $61,2 \pm 7,3$ & 20 & $60,4 \pm 5,4$ & 04 & 0,345 \\
Grupo II & $64,4 \pm 7,8$ & 22 & - & 02 & NA \\
Grupo III & $61,5 \pm 5,1$ & 09 & $63,7 \pm 4,4$ & 10 & 0,463 \\
\hline
\end{tabular}

NA: não analisável; DPE: desnutrição proteico energética

\section{Discussão}

A PA aumenta progressivamente com a idade, inicia-se com valor de PAS abaixo de $100 \mathrm{mmHg}$, em crianças menores de seis anos, até chegar ao valor de $120 / 80 \mathrm{mmHg}$ na idade adulta. Considerada como ótima ${ }^{(12)}$, essa variação pode ser entendida como uma adaptação fisiológica ao desenvolvimento físico. Na criança, a PA varia crescentemente com a idade e estudos mostram que, para isso ocorrer, deve haver também aumento do peso e da estatura ${ }^{(15,16)}$.

Os resultados obtidos quanto ao estado antropométrico no presente estudo se assemelham à literatura, a qual descreve que pacientes com anemia falciforme podem apresentar, a partir de dois anos de idade, um retardo de crescimento somático que afeta mais o peso do que a altura, acentuando-se progressivamente até os 18 anos $^{(17)}$. Já os valores das médias de PAS e PAD são similares aos obtidos nos trabalhos realizados em crianças saudáveis por Brandão ${ }^{(18)}$, Moura et a $l^{(19)}$, Sarni $e t$ a $l^{(20)}$.

Martorell et al ${ }^{(21)}$, avaliando a PA em crianças sem hemoglobinopatia, relataram que aquelas com DPE apresentavam níveis pressóricos mais baixos em relação aos de crianças eutróficas, entretanto isto não foi observado neste estudo. Cabe ressaltar que o número de pacientes com DPE da presente amostra é pequeno, não conferindo poder na análise. Diversos estudos ${ }^{(6-10)}$ realizados em adultos relataram que a DF está associada a menores níveis pressóricos e a menor incidência de HA em relação à prevalência da população geral, o que poderia ser um fator benéfico, uma vez que a ocorrência de HA é considerada como risco para crises de falcização, hipertensão pulmonar e acidente vascular isquêmico ${ }^{(11)}$.

Observou-se neste trabalho que a PA foi anormal (hipertensão e pré-hipertensão) em 14,3\% dos pacientes, 
semelhante ao observado por Becton et al ${ }^{(5)}$ em estudo com 90 pacientes portadores de DF com idades entre dois e 18 anos. A detecção de $5,7 \%$ de pacientes com HA é similar também ao relatado em trabalhos realizados com adultos e crianças portadoras de DF e também aos valores observados na literatura nacional e internacional com crianças e adolescentes saudáveis ${ }^{(4,17,19,22)}$. Cabe observar que, no estudo de Becton et al ${ }^{(5)}$, dez pacientes $(11,1 \%)$ apresentavam hipertensão, sendo que, dentre eles, quatro também tinham microalbuminúria.

A detecção de pacientes com pré-hipertensão deve ser considerada um fator de alerta, uma vez que contam com maior risco de evoluir para $\mathrm{HA}^{(23)}$, o que ocorreu em $8,6 \%$ dos pacientes da amostra aqui avaliada. Becton et a $l^{(5)}$ também encontraram percentual semelhante $(6,2 \%)$, sendo que um paciente já apresentava microalbuminúria.

Estudos publicados em crianças relacionam a $\mathrm{HA}$ ao aumento do $\mathrm{IMC}^{(23-25)}$. Essa associação não foi encontrada neste estudo provavelmente porque o IMC se manteve dentro da normalidade e inalterado em todos os grupos, entretanto pode-se supor que outros fatores estejam

\section{Referências bibliográficas}

1. Cançado RD, Jesus JA. Sickle cell disease in Brazil. Rev Bras Hematol Hemoter 2007;29:204-6.

2. Brasil. Agência Nacional de Vigilância Sanitária. Manual de diagnóstico e tratamento de doenças falciformes. Brasília: ANVISA; 2002.

3. Magalhães IQ. Renal abnormalities in sickle cell disease. Rev Bras Hematol Hemoter 2007;29:279-84.

4. Gurkan S, Scarponi KJ , Hotchkiss H, Savage B, Drachtman R. Lactate dehydrogenase as a predictor of kidney involvement in patients with sickle cell anemia. Pediatr Nephrol 2010;25:2123-7.

5. Becton LJ, Kalpatthi RV, Rackoff E, Disco D, Orak JK, Jackson SM et al. Prevalence and clinical correlates of microalbuminuria in children with sickle cell disease. Pediatr Nephrol 2010;25:1505-11.

6. Johnson CS. Arterial blood pressure and hypervicosity in sickle cell disease. Hematol Oncol Clin North Am 2005;19:827-37.

7. Johnson CS, Giorgio AJ. Arterial blood pressure in adults with sickle cell disease. Arch Intern Med 1981;141:891-3.

8. Grell GA, Alleyne GA, Serjeant GR. Blood pressure in adults with homozygous sickle cell disease. Lancet 1981;2:1166.

9. Pegelow $\mathrm{CH}$, Colangelo L, Steinberg M, Wright EC, Smith J, Phillips G et al. Natural history of blood pressure in sickle cell disease: risks for stroke and death associated with relative hypertension in sickle cell anemia. Am J Med 1997; 102:171-7.

10. Karayaylalí I, Onal M, Yíldízer K, Seyrek N, Paydas S, Akoglu E et al. Low blood pressure, decreased incidence of hypertension, and renal cardiac, and autonomic nervous system functions in patients with sickle cell syndromes. Nephron 2002;91:535-7.

11. Gordeuk VR, Sachdev V, Taylor JG, Gladwin MT, Kato G, Castro OL. Relative systemic hypertension with sickle cell disease is associated with risk of pulmonary hypertension and renal insufficiency. Am J Hematol 2008;83:15-8. envolvidos, uma vez que é reconhecido que os pacientes falcêmicos, devido ao processo vaso-oclusivo, apresentam alterações na renina plasmática, endotelina e nos metabólitos do óxido nítrico, que alteram o equilíbrio entre vasodilatação e vasoconstrição, situação não encontrada nas crianças desnutridas ${ }^{(26-28)}$.

A ocorrência de HA nas crianças e adolescentes com DF demonstra a importância da medida da PA, uma vez que pacientes que apresentam níveis pressóricos mais elevados, em relação aos valores médios, também têm maior risco de crises vaso-oclusivas e óbito.

Pode-se concluir que os valores das médias de PAS e PAD do presente estudo foram semelhantes aos obtidos nos estudos realizados em crianças saudáveis, sem hemoglobinopatia. A detecção de PA anormal (hipertensão e pré-hipertensão) em pacientes portadores de DF confirma a importância da medida rotineira da PA na prática pediátrica. Estudos multicêntricos devem ser realizados para avaliar o valor da PA em crianças e adolescentes portadores de DF e detectar as possíveis causas de hipertensão arterial nesses pacientes.

12. Sociedade Brasileira de Cardiologia; Sociedade Brasileira de Hipertensão; Sociedade Brasileira de Nefrologia. V Brazilian Guidelines in Arterial Hypertension. Arq Bras Cardiol 2007;89:e24-79.

13. World Health Organization. WHO child growth standards: methods and development. Length/height-for-age, weight-for-age, weight-for-length, weightfor-height and body mass index-for-age. Geneva: WHO; 2006.

14. World Health Organization. WHO child growth standards: methods and development. Lenth/height-for-age,weight-for-age,weight-for-length, weightfor-height and body mass index-for-age. Geneva: WHO; 2007. Available from: http://www.who.int/childgrowth/standards/technical_report/en/

15. Sinaiko AR, Gomez-Marin O, Prineas RJ. "Significant" diastolic hypertension in pre-high school black and white children. The children and adolescents blood pressure program. Am J Hypertens 1988;1:178-80.

16. De Swiet M. The epidemiology of hypertension in children. Br Med Bull 1986;42:172-5.

17. Zago MA, Kerbauy J, Souza HM, Figueiredo MS, Costa FF, Cruz SM et al. Growth and sexual maturation of Brazilian patients with sickle cell diseases. Trop Geogr Med 1992;44:317-21.

18. Brandão AP. The importance of physical development in the behavior of the arterial pressure curve in children from 6 to 9. Arq Bras Cardiol 1987;48:203-9.

19. Moura AA, Silva MA, Ferraz MR, Rivera IR. Prevalence of high blood pressure in children and adolescents from the city of Maceió, Brazil. J Pediatr ( Rio J) 2004;80:35-40.

20. Sarni RO, Souza FI, Pitta TS, Fernandez AP, Hix S, Fonseca FA. Low birth weight: influence on blood pressure, body composition and anthropometric indexes. Arq Med ABC 2005;30:76-82.

21. Martorell R, Yarbrough C, Klein RE, Lechtig A. Malnutrition, body size and skeletal maturation: interrelationships and implications for cath-up growth. Hum Biol 1979;51:371-89. 
22. Monego ET, Jardim PC. Determinants of risk of cardiovascular diseases in schoolchildren. Arq Bras Cardiol 2006;87:37-45.

23. Chobanian AV. Prehypertension revisited. Hypertension 2006;48:812-4.

24. Sorof JM, Lai D, Turner J, Poffenbarger T, Portman RJ. Overweight, ethnicity, and the prevalence of hypertension in school-aged children. Pediatrics 2004;113:475-82.

25. Rosner B, Prineas R, Daniels SR, Loggie J. Blood pressure differences between blacks and whites in relation to body size among US children and adolescents. Am J Epidemiol 2000;151:1007-19.
26. Hatch FE, Crowe LR, Miles DE, Young JP, Portner ME. Altered vascular reactivity in sickle hemoglobinopathy. A possible protective factor from hypertension. Am J Hypertens 1989;2:2-8.

27. Hammerman SI, Kourembanas S, Conca TJ, Tucci M, Brauer M, Farber HW. Endothelin-1 production during the acute chest syndrome in sickle cell disease. Am J Respir Crit Care Med 1997;156:280-5.

28. Reiter CD, Gladwin MT. An emerging role for nitric oxide in sickle cell disease vascular homeostasis and therapy. Curr Opin Hematol 2003;10:99-107. 